\begin{tabular}{|c|l|}
\hline Title & Deuterium retention and desorption behavior in oxidized ferritic steel \\
\hline Author(s) & Y amauchi, Y.; Hirohata, Y.; Hino, T. \\
\hline Citation & $\begin{array}{l}\text { Journal of Nuclear Materials, 363-365, 984.988 } \\
\text { https://doi.org/10.1016/.jnucmat.2007.01.136 }\end{array}$ \\
\hline Issue Date & 2007-06-15 \\
\hline Doc URL & http://hdl.handle.net/2115/28419 \\
\hline Type & article (author version) \\
\hline File Information & JNM363-365.pdf \\
\hline
\end{tabular}

Instructions for use 
/10/Paper Number: P3-90

\title{
Deuterium retention and desorption behavior in oxidized ferritic steel
}

\author{
*Y. Yamauchi, Y. Hirohata and T. Hino \\ Laboratory of Plasma Physics and Engineering, Hokkaido University, Kita-13, Nishi-8, Kita-ku \\ Sapporo, 060-8628 Japan
}

\begin{abstract}
Deuterium retention and desorption behavior in the oxidized F82H ferritic steel were investigated. The relation between the deuterium retention and the surface state of oxidized sample was also investigated. The deuterium retained in the ferritic steel sample was desorbed in forms of $\mathrm{HD}, \mathrm{D}_{2}, \mathrm{HDO}$, and $\mathrm{D}_{2} \mathrm{O}$. Large HDO desorption at around $600 \mathrm{~K}$ was observed in the oxidized sample. The ratio of deuterium atom desorbed in form of HDO to total amount retained in the oxidized sample was larger than that in the non-oxidized sample. At high deuterium ion fluence, the amount of deuterium retained in the oxidized sample decreased with the deuterium ion fluence. Contrary, the amount of deuterium retained in the non-oxidized sample increases with the fluence. The feature of retention in the oxidized sample at high fluence is explained by the reduction of oxygen content due to the oxygen release during the irradiation.
\end{abstract}

JNM Keywords : Hydrogen and Hydrides, Ion Irradiation, Low Activation Materials, Oxides PSI-17 Keywords : Activation, Desorption, Deuterium inventory, Oxygen

PACS Number : 68.43.Vx, 79.20.Rf, 61.82.Bg, 28.52.-s

Corresponding author : Y. Yamauchi

E-mail : yamauchi@qe.eng.hokudai.ac.jp

Postal address: Laboratory of Plasma Physics and Engineering, Hokkaido University, Kita-13, Nishi-8, Kita-ku, Sapporo, 060-8628 JAPAN 


\section{Introduction}

Low activation ferritic steel, such as $\mathrm{F} 82 \mathrm{H}(8 \mathrm{Cr}-2 \mathrm{~W})$, is regarded as a candidate material for the blanket component or the first wall in the fusion reactor [1]. Various studies of this ferritic steel have been performed so far [2-5]. This ferritic steel is well oxidized by an exposure to air. It is an important issue to clarify the influence of the surface oxide layer on fuel hydrogen retention. In our previous study, the deuterium retention of the ferritic steel exposed to air for $4 \mathrm{yr}$, which had a thick oxide layer, $80 \mathrm{~nm}$, was investigated [2, 3]. In this case, a significant amount of deuterium was trapped in the oxide layer. However, the relation between the deuterium retention and the oxide layer has not been systematically investigated.

In the present study, deuterium retention and desorption behavior in the oxidized F82H ferritic steel were investigated. In addition, the relation between the deuterium retention and the surface state of oxidized layer was also investigated.

\section{Experiments}

F82H ferritic steel developed by Japan Atomic Energy Research Institute [1] was used as the sample. Before deuterium ion irradiation, the sample was mechanically polished and then degassed in vacuum at $573 \mathrm{~K}$ for $1 \mathrm{~h}$. Two kinds of the F82H samples were prepared for ion irradiation experiments. One was the oxidized ferritic steel and another was the non-oxidized ferritic steel. The oxidation was performed by exposure to oxygen gas at $573 \mathrm{~K}$ for $30 \mathrm{~min}$. The oxygen gas pressure was $0.05 \mathrm{~Pa}$. The sample was irradiated at room temperature by deuterium ions in ECR ion source [6]. The energy of deuterium ion was $1.7 \mathrm{keV}$ and the flux was $\sim 5 \times 10^{14} \mathrm{D} / \mathrm{cm}^{2} \mathrm{~s}$. The ion fluence was varied from $1 \times 10^{18} \mathrm{D} / \mathrm{cm}^{2}$ to $7 \times 10^{18} \mathrm{D} / \mathrm{cm}^{2}$. After the irradiation, the amount of retained deuterium was measured by using the technique of thermal desorption spectroscopy, TDS. The sample was heated from RT to $1073 \mathrm{~K}$ with the 
ramp rate of $0.5 \mathrm{~K} / \mathrm{s}$. The desorption rate of deuterium during the heating was quantitatively measured by using a quadrupole mass spectrometer [7]. The amount of retained deuterium was obtained by integrating the time dependencies of the desorption rates of gases containing D. The depth profiles of elemental compositions before and after the irradiation were examined by Auger electron spectroscopy. Modification of surface morphology of the samples due to the ion irradiation and/or the oxidation was observed by a scanning electron microscope, SEM.

\section{Results and Discussion}

The elemental depth profiles in the non-oxidized sample and the oxidized sample are shown in figs.1 (a) and (b), respectively. A very thin impurity layer (1-2 nm) was observed on the surface of the non-oxidized sample. It is seen that this layer is enriched by oxygen. At the same time, $\mathrm{Fe}, \mathrm{Cr}$, and $\mathrm{C}$ concentrations are below the respective levels in the bulk. In the oxidized sample, the oxide layer with the thickness of 50 nm was formed. In this oxide layer, we observed a very low carbon content. This carbon concentration in the oxide layer was lower than that in the F82H sample exposed to air for 4 yr [3].

The elemental depth profiles in the oxidized sample after the irradiations with the fluences of $1 \times 10^{18}, 5 \times 10^{18}$, and $7 \times 10^{18} \mathrm{D} / \mathrm{cm}^{2}$ are shown in figs.2 (a), (b) and (c), respectively. The oxygen concentration in the oxide layer gradually decreased when the deuterium ion fluence increased. It is noted that oxygen depletion took place over the whole thickness of the oxide layer. These results indicate that oxygen release as $\mathrm{HDO}\left(\mathrm{D}_{2} \mathrm{O}\right)$ as well as ion induced redistribution of oxygen over the depth takes place during deuterium ion irradiation. The increase of carbon concentration around the surface may be due to the contamination by an air venting. By SEM observation, we found that the surface morphology became rough after the oxidation. The roughness increased after the ion irradiation, and then the fine protuberances 
were observed at the surface of the oxidized sample. Contrary, no dominant protuberance in the non-oxidized sample was observed at the surface for both before and after the irradiation. No significant change in the surface morphology after the irradiation was observed for the non-oxidized sample.

The deuterium retained in the ferritic steel sample desorbed in forms of $\mathrm{HD}, \mathrm{D}_{2}, \mathrm{HDO}$, and $\mathrm{D}_{2} \mathrm{O}$ during TDS measurement. Fig. 3 shows the thermal desorption spectra of these gases from the non-oxidized sample after the deuterium ion irradiation. The deuterium ion fluence was $5 \times 10^{18} \mathrm{D} / \mathrm{cm}^{2}$. Large HDO and HD desorptions were observed at 600-800 K and $900 \mathrm{~K}$, respectively. The peak temperature of $\mathrm{D}_{2} \mathrm{O}$ was similar to that of HDO, though the desorption peak was very broad.

Figs.4 (a), (b) and (c) show the thermal desorption spectra of gases containing D from the oxidized sample after the deuterium ion irradiation. The deuterium ion fluences in figs.4(a), (b) and (c) were $1 \times 10^{18}, 5 \times 10^{18}$, and $7 \times 10^{18} \mathrm{D} / \mathrm{cm}^{2}$, respectively. Large HDO desorption at around $600 \mathrm{~K}$ was observed in figs.4. The ratio of deuterium desorbed in form of HDO to the total amount of deuterium retained in the oxidized sample was significantly larger than that in the non-oxidized sample. Contrary, the ratio of deuterium desorbed in form of HD in the oxidized sample was smaller than that in the non-oxidized sample.

Fig.5 shows the ion fluence dependence of the amount of deuterium in TDS. One can conclude from this experiment that the amount of deuterium retained in the oxidized sample decreased with increase of the ion fluence. This agrees with observations of TDS of various gases desorbed from the oxidized sample in figure 4. Contrary, as it was found in the previous study, the amount of deuterium retained in the non-oxidized sample gradually increased with the deuterium ion fluence [2, 3]. These results suggest that the amount of retained deuterium becomes large when plenty of oxygen content is existed. Decrease of deuterium retention with deuterium ion fluence correlates with decrease of oxygen content. 
One may suggest that the implanted deuterium provokes oxygen release and the decrease of oxygen content provokes decrease of the deuterium trapping efficiency. One may also suggest that the deuterium content in the oxidized sample increases with the deuterium ion fluence if there was plenty of oxygen content in the oxide layer, and then deuterium interacts with oxygen giving release as HDO during deuterium implantation, resulting in the decrease of the deuterium retention. This interaction can be provoked either by high radiation damage or by ion mixing in the oxide layer.

The amount of deuterium retained in the non-oxidized sample was larger than that in the oxidized sample at high fluence, as shown in fig.5. There might be the significant difference in temperature rise at the surface during the irradiation between the oxidized and the nonoxidized samples. Thermal conductivity of the oxide layer should be different from that of F82H. Furthermore, there might be the impurity effects such as carbon on the deuterium retention in the oxidized sample. Further studies are necessary to clarify the reason of the difference in the amount of retained deuterium at high fluence region.

\section{Summary}

Deuterium retention and desorption behavior in the oxidized $\mathrm{F} 82 \mathrm{H}$ ferritic steel after deuterium ion irradiation was investigated. The oxidation was conducted by the oxygen exposure with the pressure of $0.05 \mathrm{~Pa}$ at $573 \mathrm{~K}$. After the oxidation, the oxide layer with the thickness of $\sim 50 \mathrm{~nm}$ was formed at the surface. The oxygen content gradually decreased with the increase of the deuterium ion fluence due to oxygen release as $\mathrm{HDO}\left(\mathrm{D}_{2} \mathrm{O}\right)$ during the irradiation. The deuterium retained in the oxidized sample desorbed in forms of $H D, D_{2}$, HDO and $\mathrm{D}_{2} \mathrm{O}$ during TDS measurement. Mainly HDO desorbed with the peak at around 600 $\mathrm{K}$ from the oxidized sample. The contribution of HDO desorption becomes relatively large, while the contribution of HD desorptions becomes small, compared with the non-oxidized 
sample. The amount of deuterium retained in the oxidized sample decreases with deuterium ion fluence contrary to the increasing dependence for the non-oxidized sample. These dependences may cross each other. The feature of retention in the oxidized sample may be connected with D-O interaction during deuterium ion implantation. 


\section{References}

[1] K. Shiba, A. Hishinuma at al., Properties of low activation ferritic steel F82H IEA Heat, JAERI-Tech 97-038 (1997). (in Japanese)

[2] H. Ogawa, Y. Yamauchi et al, "Impurity Release and Deuterium Retention Properties of a Ferritic Steel Wall in JFT-2M”, J. Nucl. Mater., 329-333 (2004) 678.

[3] T. Hino, K. Yamaguchi et al, "Deuterium retention and physical sputtering of low activation ferritic steel”, Plasma Science \& Technology, 7 (2005) 2737.

[4] H. Kimura, M. Sato, H. Kawashima, et al., Progress of advanced material tokamak experiment (AMTEX) program on JFT-2M, Fusion Eng. and Design 56-57 (2001) 837.

[5] K. Tsuzuki, M. Sato, H. Kawashima, et al., Recent activities on the compatibility of the ferritic steel wall with the plasma in the JFT-2M tokamak, J. Nucl. Mater. 307-311 (2002) 1386.

[6] Y. Yamauchi, T. Hino et al, J. Nucl. Mater., 241-243 (1997) 1016.

[7] Y. Nakayama, S. Fukuda and T. Yamashina, J. Vac. Soc. Jpn., 32 (1989) 415. (in Japanese) 
Figure captions

Fig.1 Depth profiles of basic elements for, (a) the non-oxidized sample and (b) the oxidized sample before the deuterium ion irradiation.

Fig.2 Depth profiles of basic elements for the oxidized sample after the deuterium ion irradiation. The deuterium ion fluences were (a) $1 \times 10^{18}$, (b) $5 \times 10^{18}$, and (c) $7 \times 10^{18}$ $\mathrm{D} / \mathrm{cm}^{2}$, respectively.

Fig.3 Thermal desorption spectra of gases desorbed from the non-oxidized sample after the deuterium ion irradiation.

Fig.4 Thermal desorption spectra of deuterium-contained gases from the oxidized sample after deuterium ion irradiation. The deuterium ion fluences were (a) $1 \times 10^{18}$, (b) $5 \times 10^{18}$, and (c) $7 \times 10^{18} \mathrm{D} / \mathrm{cm}^{2}$, respectively.

Fig.5 Deuterium fluence dependence of the amount of retained deuterium. 
(a)

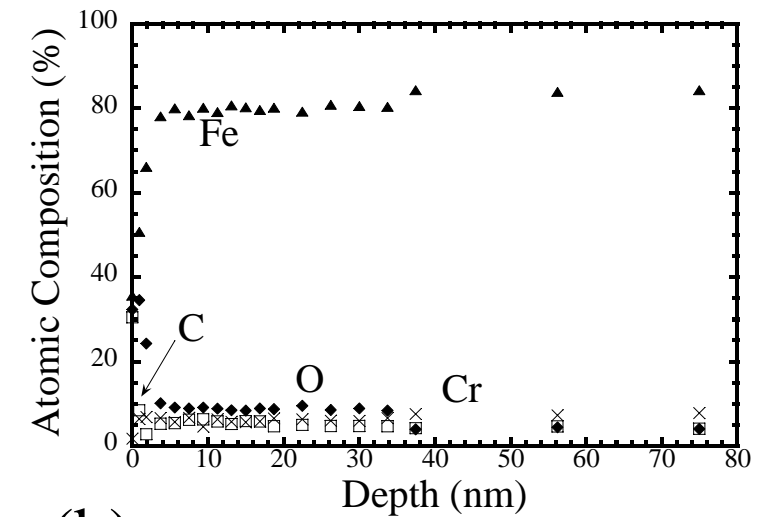

(b)

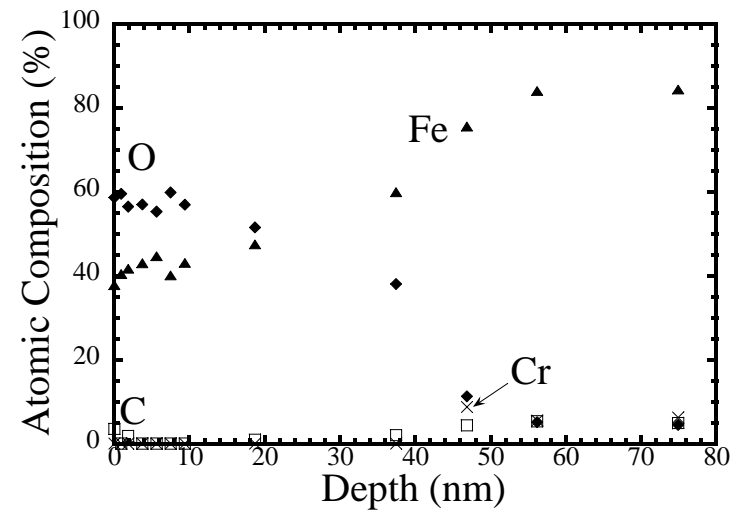

Fig.1 
(a)

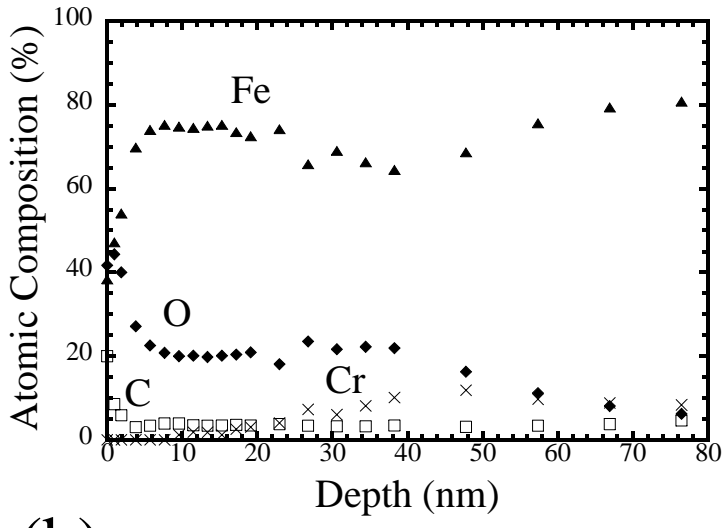

(b)

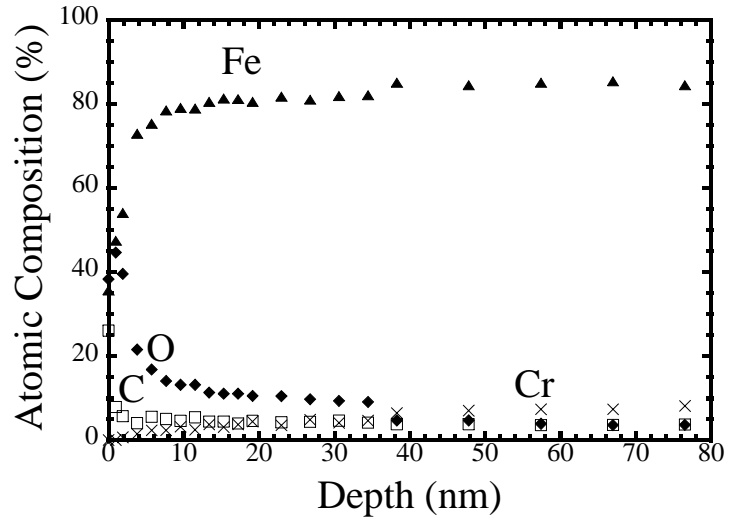

(c)

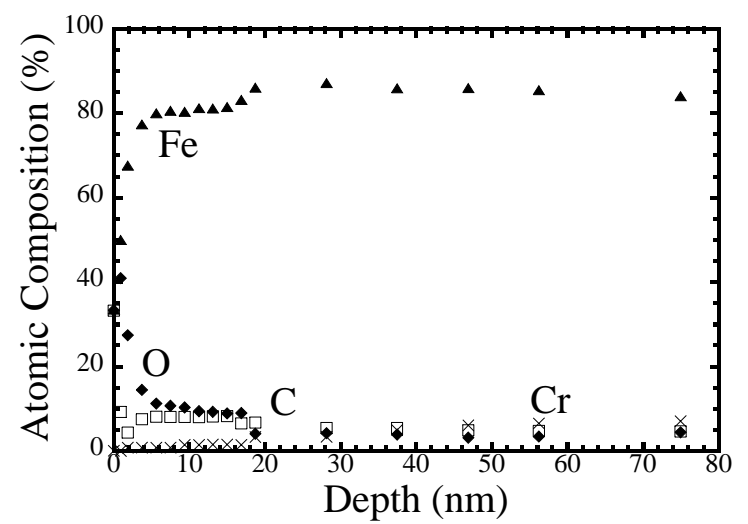

Fig.2 


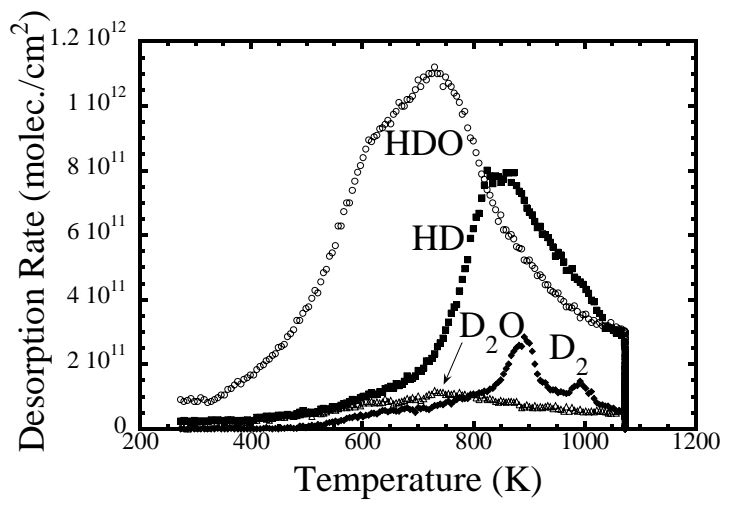

Fig.3 
(a)

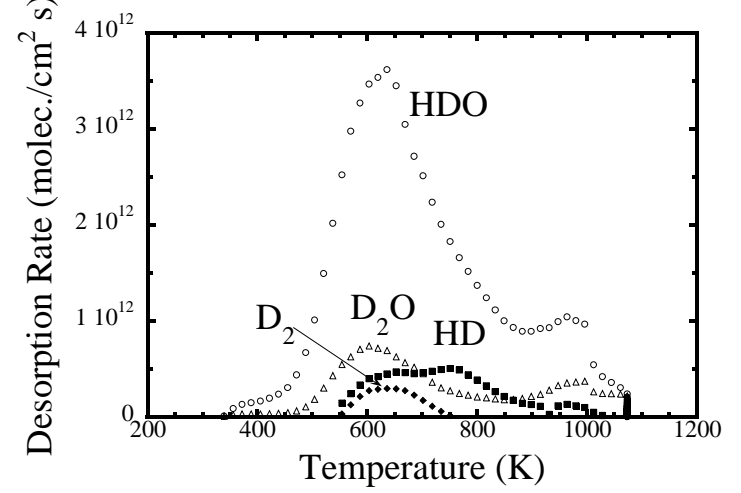

(b)

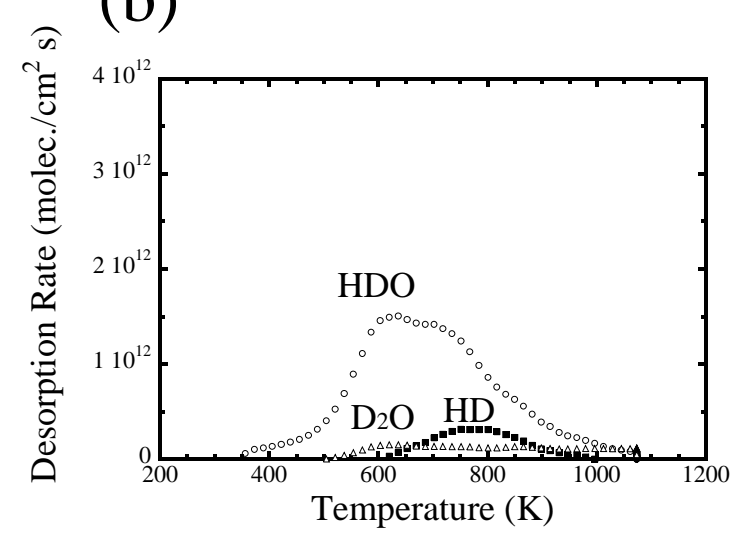

(c)

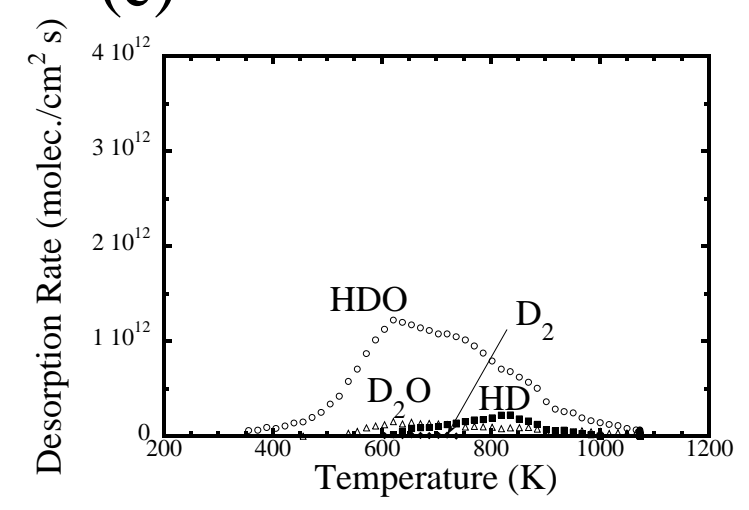

Fig.4 


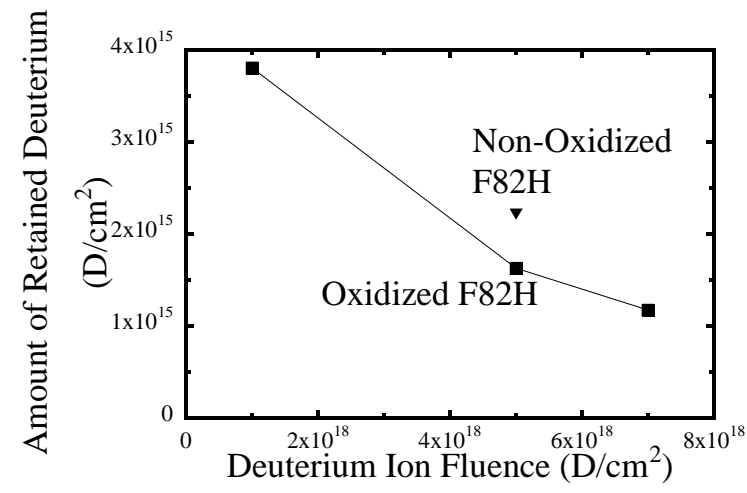

Fig.5 